P55 (continued)

Conclusions and Implications: Other attempts to measure community readiness for change have been explored, but no quantitative research methods for assessing a community's readiness for health and wellness change have been developed. A psychometrically sound and validated survey offers the possibility of accurate measuring community readiness for supporting successful institution of health and wellness interventions.

Funding: USDA

\section{P56 Development of the FoodCorps Healthy School Progress Report to Measure School Food Programming and Environment}

Pamela Koch, EdD, RD, pak14@tc.columbia.edu, Columbia University, 525 West 120th Street, Box137, New York, NY 10027; Randi Wolf, PhD, MPH, Columbia University; Matthew Graziose, PhD, Chobani; Raynika Trent, MS, MEd, Columbia University; Heewon Lee Gray, PhD, RD, University of South Florida; Isobel Contento, PhD, Columbia University

Objective: To develop a comprehensive tool to measure nutrition education, school garden experiences, healthy school meals, and schoolwide culture of health by revising a less comprehensive one.

Study Design, Setting, Participants: Collect process and outcome data and revise for 5 total versions. FoodCorps schools across United States (2014-15 and 2015-16 school years). FoodCorps service members, service site supervisors, school personnel, and stakeholders.

Outcome Measures and Analysis: Version 1: informed by literature review, interviews with 7 service members, and feedback from FoodCorps staff and board. Version 2: informed by 3 focus group phone calls and written comments from 35 stakeholders, including 6 school-based nutrition education researchers. Version 3: showed 11 high implementation schools had high HSPR scores and 9 "emerging" schools had low HSPR scores. Version 4: showed change scores for 298 FoodCorps schools completing the HSPR (Fall 2015 and Spring 2016). Conducted cognitive testing and process interviews with 9 FoodCorps Schools (3 people from each), analyzed for emerging themes. Version $5 \&$ Final HSPR: Incorporated results from prior versions.

Results: The HSPR is evidence-informed, has content and construct validity, and is sensitive to change. The tool measures FoodCorps programming in three areas of service: Hands-on Learning, Healthy School Meals, and Schoolwide Culture of Health. Average baseline scores were 42.7 (out of 100) and increasedeight points over one year. Schools that started with lower scores had bigger change scores.

Conclusions and Implications: The HSPR is a comprehensive measure of a healthy school environment and can be used by FoodCorps and other school-based nutrition education programs to assess levels of programming.

Funding: FoodCorps Inc.

\section{P57 Environmental Barriers to Healthful Dietary and Physical Activity Behaviors in the EFNEP Participant Population}

Lauren Rhoades, BS, MBA, lauren.rhoades@colostate.edu, Colorado State University, 502 West Lake Street, Fort Collins, CO 80526; Garry Auld, PhD, Colorado State University; Susan Baker, EdD

Objective: Direct education programs, including the Expanded Food and Nutrition Education Program (EFNEP), target personal barriers to enable behavior change. However, an opportunity exists to pursue a holistic policy, systems, and environment approach by targeting some environmental barriers (limited availability, high cost, safety, lack of transportation, and challenges caused by poor weather). The Western Regional Nutrition Education and Obesity Prevention Center of Excellence initiated a study to gather perspectives of EFNEP paraprofessional educators on how environmental barriers affect EFNEP participants and environmental changes which could be made to encourage more healthful participant behaviors.

Study Design, Setting, Participants: Ten telephone focus groups were held with fifty EFNEP educators from ten states during April and May of 2016.

Outcome Measures and Analysis: Focus groups were audio-recorded, transcribed, and independently coded by two reviewers to identify recurring themes.

Results: Educators provided valuable anecdotal evidence regarding environmental barriers and generated potential solutions for specific barriers based on examples from their communities. For example, potential solutions to the availability barrier include creating mobile farmers' markets, partnering with volunteer groups to provide free food delivery from food banks or retailers to areas with limited access, working with alternative retailers to offer produce in urban areas, providing seeds to encourage home gardening, or facilitating development of or participation in community gardens.

Conclusions and Implications: Results will guide additional research and inform development of environmental support activities for direct education programs reaching low-income participants. Activities will aim to empower participants to change environmental barriers in their micro-environments.

Funding: None

\section{P58 Good Choice Healthier Retail Initiative: Increasing Access and Appeal of Healthy Items in Rural Alabama}

Cecilia Tran, BS, Alabama Extension at Auburn University; Alicia Powers, PhD, arp0042@auburn.edu, Alabama Extension at Auburn University, 206 Duncan Hall, Auburn University, AL 36849; Barb Struempler, PhD, Alabama Extension at Auburn University; Sondra Parmer, PhD; Katie Funderburk, RD, MS 